\title{
Reinjection of thallium for detection of viable myocardium: why not do it immediately?
}

Patients with undiagnosed chest pain referred for myocardial thallium-201 stress perfusion scintigraphy can largely be divided into two groups-those with suspected coronary artery disease and those with known coronary artery disease. Those with known coronary artery disease can be subdivided into those with coronary artery disease identified by coronary arteriography and those with documented myocardial infarction. For each of the three subgroups there is a different clinical question. In patients with suspected coronary artery disease the major question is the absence or presence of myocardial ischaemia. In patients without prior myocardial infarction but with angiographically confirmed coronary artery disease, the predominant question is-apart from whether or not ischaemia is present-the site of myocardial ischaemia. In patients with myocardial infarction, the distinction between reversible ischaemia and scar tissue is important. The isolated question of myocardial viability is only relevant when residual viability is suspected in severe dysfunctional myocardial regions-that is, where there is stunning or hibernation. Revascularisation procedures are warranted only if viability is demonstrated.

In scintigraphic terms, myocardial viability is determined by the absolute amount of tracer uptake in the dysfunctional region or by defect reversibility after stress. ${ }^{1}$ The additional information on ischaemia is clinically useful and therefore stress protocols should be used if possible. ${ }^{2}$ Reinjection of thallium-201 after conventional stress 3-4 $\mathrm{h}$ redistribution imaging has become a standard procedure in many hospitals (figure). With this approach myocardial viability is detected in about $50 \%$ of segments showing persistent defects at redistribution imaging and these results resemble those of positron emission tomography. ${ }^{3-8}$ Because scintigraphy is cheaper ${ }^{9}$ various protocols using thallium-201 should be tested before proceeding to more complicated and or sophisticated procedures such as positron emission tomography..$^{10-12}$ In a study from our department using the standard approach to thallium-201 reinjection, reinjection of thal- lium after 3-4 hours' redistribution showed defect reversibility (and thus viability) in $46 \%$ of segments initially classified as persistent (scar tissue). ${ }^{13}$ The requirement for an additional set of images after redistribution is a major drawback of the standard protocol. It prolongs the imaging time by about an hour. In a subsequent study from our institution, immediate reinjection of thallium-201 after acquisition of the stress images followed by imaging 60 minutes later yielded promising results in the detection of myocardial viability, ${ }^{14}$ and reduced the total investigation time to a maximum of 2.5 hours (figure). We compared our results in these two studies. ${ }^{1314}$ The standard reinjection approach showed total defect reversibility in a similar proportion of segments as the immediate reinjection approach $(70 \% v 69 \%)$, and moreover, myocardial viability was observed in a similar percentage of patients ( $88 \% v 91 \%$, respectively).

Two recent studies on immediate reinjection imaging support our findings. ${ }^{15} 16$ Wackers et al compared the results of the standard approach and the immediate reinjection approach in the same group of 45 patients: agreement for defect reversibility was excellent (36\% v 34\%, respectively). Galli and Marcassa showed that immediate thallium-201 reinjection was a good method for assessing myocardial viability in 120 patients with left ventricular dysfunction.

Underwood and Pennell have commented on the practical implications of the standard reinjection approachthe uncertainty about whether an additional dose of thallium is needed and the extra imaging time required. Is reinjection necessary when 3-4 hour redistribution imaging might answer some of the clinical questions? Their comments certainly apply to the standard reinjection approach. None the less, withholding thallium reinjection in patients who have reversible defects at 3-4 hour redistribution imaging requires an accurate on-line analysis (visual and quantitative) immediately after acquisition of the redistribution images, and when the defect persists the patient will need a subsequent injec-
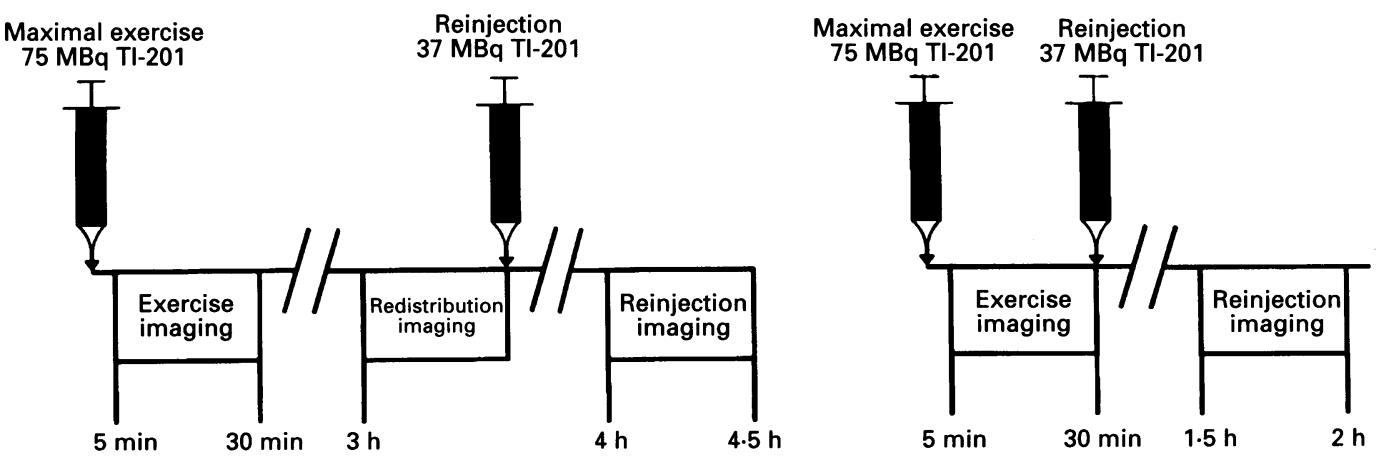
tion and extension of the imaging procedure, which may interfere with a busy clinical schedule. Though it is true that if the patient only shows reversible defects at redistribution it may save the second injection of thallium, the remaining allocated camera time will probably be unused. This is not cost-effective. Even if the redistribution images without reinjection provide sufficient information, the total imaging procedure still takes at least 4 hours. Immediate reinjection avoids the uncertainty about an additional dose (because all patients are reinjected) and considerably reduces total imaging time $(2 \cdot 5$ hours). Underwood and Pennell also referred to the problem of the unnecessary high radiation burden. However, the issue of a relatively high radiation dose is inherent in the choice of thallium as the radionuclide. If reduction of radiation dose is the goal, the use of technetium-labelled imaging agents should be considered, especially in patients in whom the detection of coronary artery disease itself is the primary clinical issue rather than the assessment of viability in dysfunctional myocardial regions.

\section{Departments of Nuclear Medicine and Cardiology,}

FERTHE L F VAN ECK-SMIT University Hospital Leiden

Rijnsburgerweg 10

2333 AA Leiden,

The Netherlands.

1 Ragosta M, Beller GA, Watson DD, Kaul S, Gimple LW. Quantitative planar rest-redistribution thallium-201 imaging in detection of myocardial viability and prediction of improvement in left ventricular function after coronary bypass surgery in patients with severely depressed left ventricular function. Circulation 1993;87:1630-41.

2 Dilsizian V, Perrone-Filardi P, Arrighi JA, Bacharach SL, Quyyumi AA, Freedman NMT, et al. Concordance and discordance between stressredistribution-reinjection and rest-redistribution thallium imaging for assessing viable myocardium; comparison with metabolic activity by positron emission tomography. Circulation 1993;88:941-52.

3 Dilsizian V, Rocco TP, Freedman NMT, Leon MB, Bonow RO Enhanced detection of ischemic but viable myocardium by the reinjection of thallium after stress-redistribution imaging. N Engl F Med 1990; 323:141-6.

4 Rocco TP, Dilsizian V, McKusick KA, Fischman AJ, Boucher CA, Strauss HW. Comparison of thallium redistribution with rest "reinjection" imaging for the detection of viable myocardium. $\mathrm{Am}$ f Cardiol 1990;66:158-63.

5 Bonow RO, Dilsizian V, Cuocolo A, Bacharach SL. Identification of viable myocardium in patients with chronic coronary artery disease and left ventricular dysfunction. Comparison of thallium scintigraphy with left ventricular dysfunction. Comparison of thallium scintigraphy with
reinjection and PET imaging with $18 \mathrm{~F}$-fluorodeoxyglucose. Circulation reinjection and

6 Tamaki N, Ohtani H, Yamashita K, Magata Y, Yonekura Y, Nohara R, et al. Metabolic activity in the areas of new fill-in after thallium-201 reinjection: comparison with positron emission tomography using fluorine-18-deoxyglucose. F Nucl Med 1991;32:673-8.

7 Perrone-Filardi P, Bacharach SL, Dilsizian V, Maurea S, Marin-Neto JA, Arrighi JA, et al. Metabolic evidence of viable myocardium in regions with reduced wall thickness and absent wall thickening in patients with chronic ischemic left ventricular dysfunction. $\mathcal{F}$ Am Coll Cardiol 1992; 20:161-8.

8 Perrone-Filardi P, Bacharach SL, Dilsizian V, Maurea S, Frank JA Bonow RO. Regional left ventricular wall thickening: relation to regional uptake of 18 -fluorodeoxyglucose and thallium-201 in patients regional uptake of 18-fluorodeoxyglucose and thallium-201 in patients
with chronic coronary artery disease and left ventricular dysfunction. with chronic coronary artery
Circulation 1992;86:1125-37.

9 Zaret BL, Wackers FJ. Nuclear cardiology (first of two parts). $N$ Engl f Med 1993;329:775-83.

10 Iskandrian AS. Thallium reinjection imaging: the search for an optima protocol. $\mathcal{F}$ Nucl Med 1993;34:743-5.

11 Underwood SR, Pennell DJ. Viable myocardium and reinjection of thallium. Br Heart f 1992;68:537-9.

12 Wackers FJ. The maze of myocardial perfusion protocols in 1994. $\mathcal{F ~ N u c l}$ Cardiol 1994;1:180-8.

13 Kuijper AFM, Vliegen HW, van der Wall EE, Oosterhuis WP, Zwinderman AH, van Eck-Smit BLF, et al. The clinical impact of thalZwinderman AH, van Eck-Smit BLF, et al. The clinical impact of thal-
lium-201 reinjection scintigraphy for detection of myocardial viability. lium-201 reinjection scintigraphy

14 van Eck-Smit BLF, van der Wall EE, Kuijper AFM, Zwinderman AH, Pauwels EKJ. Immediate thallium-201 reinjection following stress imaging: A time-saving approach for detection of myocardial viability. $\mathcal{F} \mathrm{Nucl}$ Med 1993;34:737-43.

15 Wackers FJ, Pieri PL, McMahon M, Della Vittoria G, Tisselli A Moscatelli G, et al. Quantitative reproducibility of repeated plana thallium-201 stress imaging. F Nucl Med 1993;34:44P.

16 Galli M, Marcassa C. Thallium-201 redistribution after early reinjection in patients with severe stress perfusion defects and ventricular dysfunction. Am Heart $\mathcal{F}$ 1994;128:41-52. 